\title{
Incidence and Risk Factors Associated with Superior Mesenteric Artery Syndrome following Surgical Correction of Scoliosis
}

\author{
Ju Young Kim*, Hak Sun Kim*, Eun Su Moon*, Jin Oh Park*, Dong Eun Shin ${ }^{\dagger}$, \\ Gene Kyu Lee ${ }^{\ddagger}$, Jung Won Ha ${ }^{\$}$, Yeun Su Jung ${ }^{\$}$ \\ *Department of Spinal Orthopedic Surgery, Yong-Dong Severance Hospital, Yonsei University, College of Medicine, \\ Seoul, Korea, ${ }^{\dagger}$ Department of Spinal Orthopedic Surgery, Bundang Cha Hospital, Seoul, Korea, \\ ${ }^{\ddagger}$ Washington University in St. Louis, St. Louis, MO 63105, Department of Orthopaedic Surgery, \\ $\$$ National Health Insurance Corporation Ilsan Hospital
}

Study Design: Retrospective study.

Purpose: To more accurately determine the incidence and clarify risk factors.

Overview of Literature: Superior mesenteric artery syndrome is one of the possible complications following correctional operation for scoliosis. However, when preliminary symptoms are vague, the diagnosis of superior mesenteric artery syndrome may be easily missed.

Methods: We conducted a retrospective study using clinical data from 118 patients ( 43 men and 75 women) who underwent correctional operations for scoliosis between September 2001 and August 2007. The mean patient age was 15.9 years (range 9 24 years). The risk factors under scrutiny were the patient body mass index (BMI), change in Cobb's angle, and trunk length.

Results: The incidence of subjects confirmed to have obstruction was $2.5 \%$. However, the rate increased to $7.6 \%$ with the inclusion of the 6 subjects who only showed clinical symptoms of obstruction without confirmative study. The BMI for the asymptomatic and symptomatic groups were $18.4 \pm 3.4$ and $14.6 \pm 3$, respectively. The change in Cobb's angle for the asymptomatic and symptomatic groups were $24.8 \pm 13.6^{\circ}$ and $23.4 \pm 9.1^{\circ}$, respectively. The change in trunk length for the asymptomatic and symptomatic groups were $2.3 \pm 2.1 \mathrm{~cm}$ and $4.5 \pm 4.8 \mathrm{~cm}$, respectively. Differences in Cobb's angle and the change in trunk length between the two groups did not reach statistical significance, although there was a greater increase in trunk length for the symptomatic group than for the asymptomatic group.

Conclusions: Our study shows that the incidence of superior mesenteric artery syndrome may be greater than the previously accepted rate of $4.7 \%$. Therefore, in the face of any early signs or symptoms of superior mesenteric artery syndrome, prompt recognition and treatment are necessary.

Key Words: Scoliosis, Superior mesenteric artery syndrome, Body mass index, Trunk length

\section{Introduction}

Abdominal pain is one of the most common complaints in patients who have undergone correctional operations for scoliosis. However, little attention has been directed to this matter, because it is easily treatable through conservative treatment methods. One of the causes of abdominal pain following a scoliosis correctional operation is superior mesenteric artery (SMA) syndrome, which was first discussed by Rokitansky in $1842^{1}$. SMA syndrome manifests in patients approximately 1 week after operation ${ }^{2-7}$. The SMA passes in front of the third portion of the duodenum at the level of the third lumbar vertebra. Consequently, SMA

Corresponding author: Hak Sun Kim, MD

Department of Orthopedic Surgery, Yongdong Severance Hospital 612 Eonjuro, Gangnam-gu, Seoul, 135-720, Republic of Korea

Tel: +82-2-2019-3411, Fax: +82-2-573-5393, E-mail: haksunkim@yuhs.ac 
syndrome can cause duodenal obstruction, which leads to nausea, bilious vomiting, and abdominal distention. Conservative treatment measures usually resolve this disorder. However, severe complications such as electrolyte imbalance or gastric perforation, may rarely occur, potentially leading to patient death ${ }^{1,4,89}$. Therefore, a comprehensive understanding of risk factors and incidence is important for pre- and post-operative patient care, especially considering that the potential complications are life-threatening.

The association between correctional operations for scoliosis and SMA syndrome is particularly compelling, because scoliosis patients carry a greater risk for SMA syndrome.

One prominent characteristic of scoliosis patients is their low weight relative to their height. Patients who are markedly underweight, who undergo a correctional operation, and who consequently experience an increase in height or a decrease in body weight are at an even greater risk.

Various research efforts have been directed at analyzing the effects of post-operative weight and height changes on the risk of SMA syndrome ${ }^{3,6,10,11}$. It is already known that an increase in height, especially the trunk length, is one factor that increases the risk of SMA syndrome.

The accepted incidence of SMA syndrome is between $1 \%$ and $4.7 \%$. However, this rate has been determined based on studies of patients who have been confirmed to have intestinal obstruction ${ }^{2.5,7,12}$. The inclusion of patients without confirmed intestinal obstruction would undoubtedly increase this figures.

The purpose of this study was to more accurately determine the incidence by analyzing patients confirmed to have intestinal obstruction and those with less explicit symptoms, and to closely analyze the effects of post-operative height and weight changes on the risk of SMA syndrome.

\section{Materials and Methods}

Between September 2001 and August 2007, a retrospective clinical study was conducted on patients who underwent correctional operations for scoliosis at the Youngdong Severance and Bundang Cha Hospitals. The subject pool consisted of those patients who underwent posterior spinal fusion with or without anterior spinal fusion. The lower part of the fusion had to be below the second lumbar vertebrate. The study group consisted of 118 patients (43 men and 75 women) covering an age range from 9 to 24 years, with the mean age being 15.9 years. Furthermore, 80 patients had adolescent idiopathic scoliosis (AIS), and 38 had neuromuscular scoliosis (NMS) (Table 1). From this pool of patients, we selected those subjects suffering from excessive nausea, bilious vomiting, or abdominal distension after approximately one week (range five to ten weeks). Patients who showed only moderate nausea or severe nausea with no accompanying vomiting were excluded. Patients with abdominal distention underwent a simple abdominal x-ray to exclude postoperative ileus. Confirmation was restricted to those patients who showed positive results on upper gastrointestinal (GI) barium contrast study.

The risk factors analyzed in this study included body mass index (BMI), and changes in both Cobb's angle and trunk length. BMIs were calculated by dividing each patient's weight (kilograms) by the square of his or her height (meter). All values were obtained at the time of hospitalization. The BMIs of patients under 20 years of age

Table 1. Demographics of the entire subject pool according to the etiology

\begin{tabular}{lccr}
\hline & AIS $(\mathrm{n}=80)$ & NMS $(\mathrm{n}=38)$ & p-value \\
\hline Weight $(\mathrm{Kg})$ & $47.1 \pm 10.5$ & $36.9 \pm 11.5$ & $<0.05$ \\
Height $(\mathrm{cm})$ & $160.1 \pm 8.0$ & $143.5 \pm 17.1$ & $<0.05$ \\
BMI $\left(\mathrm{Kg} / \mathrm{m}^{2}\right)$ & $18.3 \pm 3.0$ & $17.9 \pm 4.7$ & 0.83 \\
Age $($ years $)$ & $15.9 \pm 3.6$ & $13.9 \pm 4.7$ & 0.04 \\
Pre-Op.Cobb's angle $\left(^{\circ}\right)$ & $41.6 \pm 11.5$ & $54.4 \pm 18.9$ & $<0.05$ \\
Post-Op. Cobb's angle $\left(^{\circ}\right)$ & $16.2 \pm 8.3$ & $31.2 \pm 18.1$ & $<0.05$ \\
Change in angle $\left(^{\circ}\right)$ & $20.7 \pm 15.2$ & $17.6 \pm 15.8$ & 0.17 \\
& $(60.2 \pm 20 \%)$ & $(42.5 \pm 23.5 \%)$ & $(0.001)$ \\
Pre-Op TL $(\mathrm{cm})$ & $40.9 \pm 3.3$ & $33.1 \pm 5.6$ & $<0.05$ \\
Post-Op TL $(\mathrm{cm})$ & $42.6 \pm 3.1$ & $37.4 \pm 5.6$ & $<0.05$ \\
Change in TL $(\mathrm{cm})$ & $0.7 \pm 1.2$ & $1.6 \pm 3.1$ & 0.08 \\
& $(4.7 \pm 4.0 \%)$ & $(14.0 \pm 12.3 \%)$ & $(0.002)$ \\
\hline
\end{tabular}

AIS: adolescent idiopathic scoliosis, NMS: neuromuscular scoliosis, Op.: operation, TL: trunk length. 
were calculated and analyzed as a percentile, as recommended by the Korean Society of Pediatrics. These values were both age- and sex-specific. The change in Cobb's angle and trunk length were analyzed using a whole spine $\mathrm{x}$-ray. The trunk length was obtained by measuring the vertical length from the point where the line connecting the apex of the first rib crosses the mid-point of the T1 vertebrae, down to the point where it meets the line connecting the two iliac crests (Fig. 1). A two-tailed student $t$ test was used to calculate the p-value. A p-value less than 0.05 was considered statistically significant.

\section{Results}

Three patients (1 AIS and 2 NMS) were confirmed to have SMA syndrome, and 6 patients (3 AIS and 3 NMS) were thought to suffer from SMA syndrome based on clinical symptoms without barium radiography confirmation (Fig. 2). The incidence of confirmed patients was $2.5 \%$ (AIS 1.3\% and NMS 5.3\%). However, with the inclusion of the 6 patients thought to have the disorder based on clinical symptoms, the incidence increased to $7.6 \%$ (AIS 5\% and NMS $13.2 \%$ ). The BMI for the asymptomatic and symptomatic group were $18.5 \pm 3.5$ (AIS 18.4 \pm 2.9 , NMS $18.7 \pm$

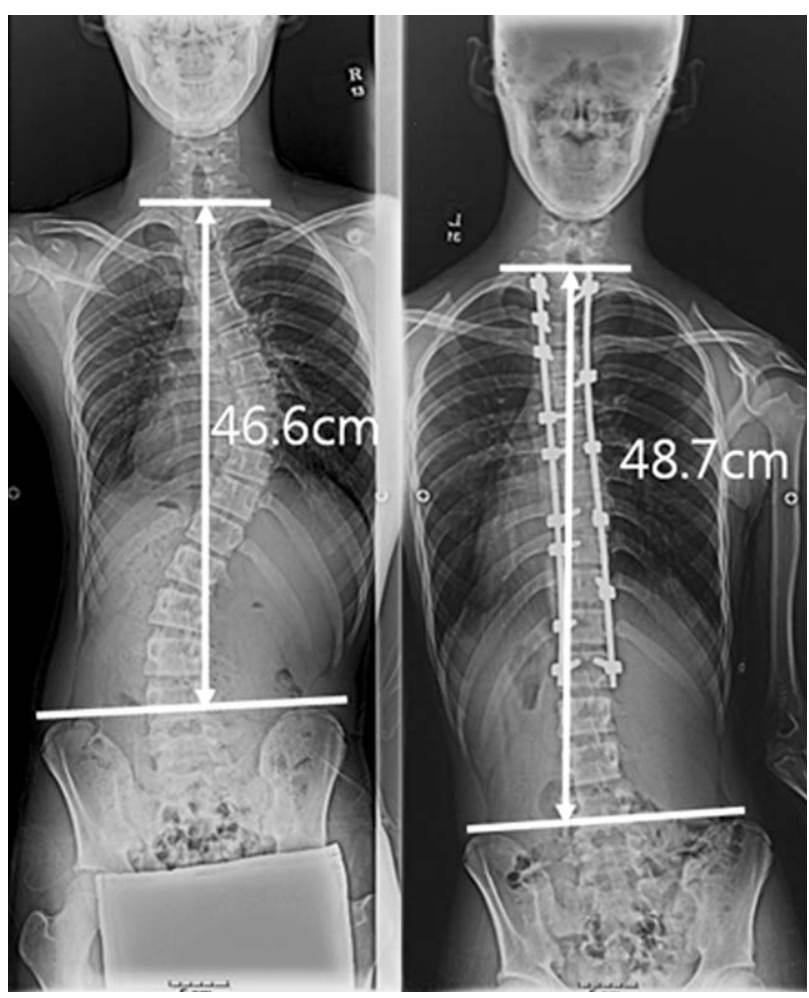

Fig. 1. Trunk length measurement.

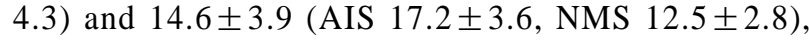
respectively, with a statistically significant difference between the groups (Tables 2-4). NMS patients showed a more significant difference in their BMI values. Analysis of age-sensitive BMI percentile data showed that 32 patients (29.4\%) were under the 5th percentile in the asymptomatic group and 6 patients (66.7\%) were under the 5th percentile in the symptomatic group. The change in Cobb's angle for the asymptomatic and symptomatic groups were $24.9 \pm$ $13.6^{\circ}$ (AIS $27.0 \pm 12.6^{\circ}$, NMS $21.8 \pm 14.6^{\circ}$ ) and $23.4 \pm$ $9.1^{\circ}$ (AIS $20.5 \pm 6.3^{\circ}$, NMS $25.8 \pm 11.0^{\circ}$ ), respectively, whereas the change in trunk length was $2.3 \pm 2.1 \mathrm{~cm}$ (AIS $1.9 \pm 1.5$, NMS $2.9 \pm 2.7$ ) for the asymptomatic group and $4.5 \pm 4.8 \mathrm{~cm}$ (AIS $0.8 \pm 0.3$, NMS $7.0 \pm 5.1$ ) for the symptomatic group. There was no statistically significant difference between the groups, although the change in trunk length for the symptomatic group was greater than that for the asymptomatic group. Of the confirmed SMA syndrome subjects, 2 showed improvement through conservative measures, while 1 subject showed a resolution in symptoms following a duodenojejunostomy (Fig. 3).

\section{Discussion}

Abdominal pain is a common complaint following correctional operation for scoliosis. However, the majority of physicians do not pay proper attention to this symptom. The satisfaction that patients and guardians have regarding operational success is based not only on the final surgical outcome, but also on the post-operative care they receive. Consequently, managing and treating abdominal pain following surgery is very important. The first task is to research and discover the cause of pain. Consequently, a comprehensive understanding of the incidence of the disorder is crucial, especially when the potential complications are life-threatening. The reported mortality rate in patients suffering from SMA syndrome is $33 \%^{13}$. The incidence of SMA syndrome is thought to be between $1 \%$ and $4.7 \%^{25,7,12}$.However, these numbers are based on patients confirmed to suffer from the disease. In this study, we considered both the confirmed patients and those with only clinical symptoms of SMA syndrome. The incidence turned out to be approximately $7.6 \%$, with the rate of SMA syndrome in patients suffering from NMS being a striking $13.2 \%$. It is also important to take into consideration the time when the disorder manifests. In this retrospective study, the subject pool was com- 

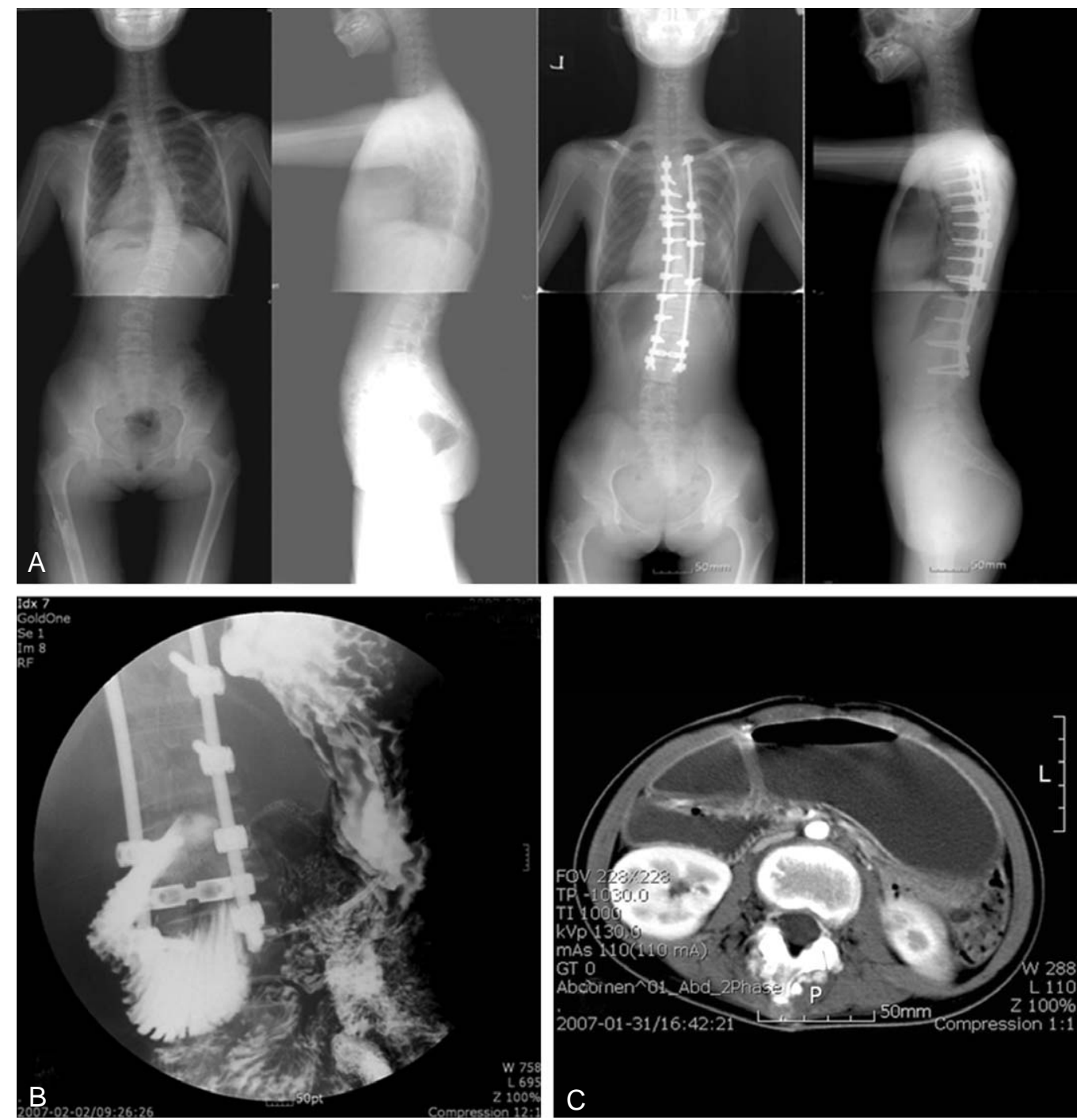

Fig. 2. A 14-year-old girl with adolescent idiopathic scoliosis. (A) Whole spine radiographs demonstrated a pre-operative Cobb angle of $30^{\circ}$ and a trunk length of $45.4 \mathrm{~cm}$. Post-operatively, the Cobb angle was $15^{\circ}$, and the trunk length was $43.6 \mathrm{~cm}$. (B) The barium contrast study confirmed the presence of SMA syndrome. (C) An abdominal CT scan showed compression of the duodenum by the SMA.

prised of subjects suffering from symptoms potentially caused by SMA syndrome, at 5 to 10 days post-operative. This mode of selection was based on previous studies showing that the symptoms from SMA syndrome manifest approximately 1 week post-operatively ${ }^{3,5,7}$. However, there have been dissenting views stating that symptoms can arise anywhere from 3 to 40 days post-operatively ${ }^{2,4,6}$. Though our subject selection was based on clinical symptoms and on a restrictive time period for the manifestation of the disorder, we showed that the incidence of SMA syndrome is more common than previously thought.

More attention is now being directed to SMA syndrome in patients undergoing correctional operations for scoliosis, because of the heightened susceptibility of these patients to the disorder ${ }^{3,610,11,14}$. The majority of scoliosis patients have a lighter body weight relative to their height ${ }^{4,67,15}$. This imbalance of height to weight is exacerbated when these patients undergo a correctional operation that abruptly increases their trunk length and thus their height, or when they fail to receive adequate post-operative care, thereby resulting in weight loss.

The primary factor we analyzed was the BMI, which provides us with a height-to-weight ratio that can be compared across individuals. Earlier studies have shown that when the age- and sex-matched weight of an individual is below the 25 th percentile, the risk of SMA syndrome is greater ${ }^{4,6,7,12,15}$. Our study showed a significant difference in the BMI between asymptomatic and symptomatic persons. Even 

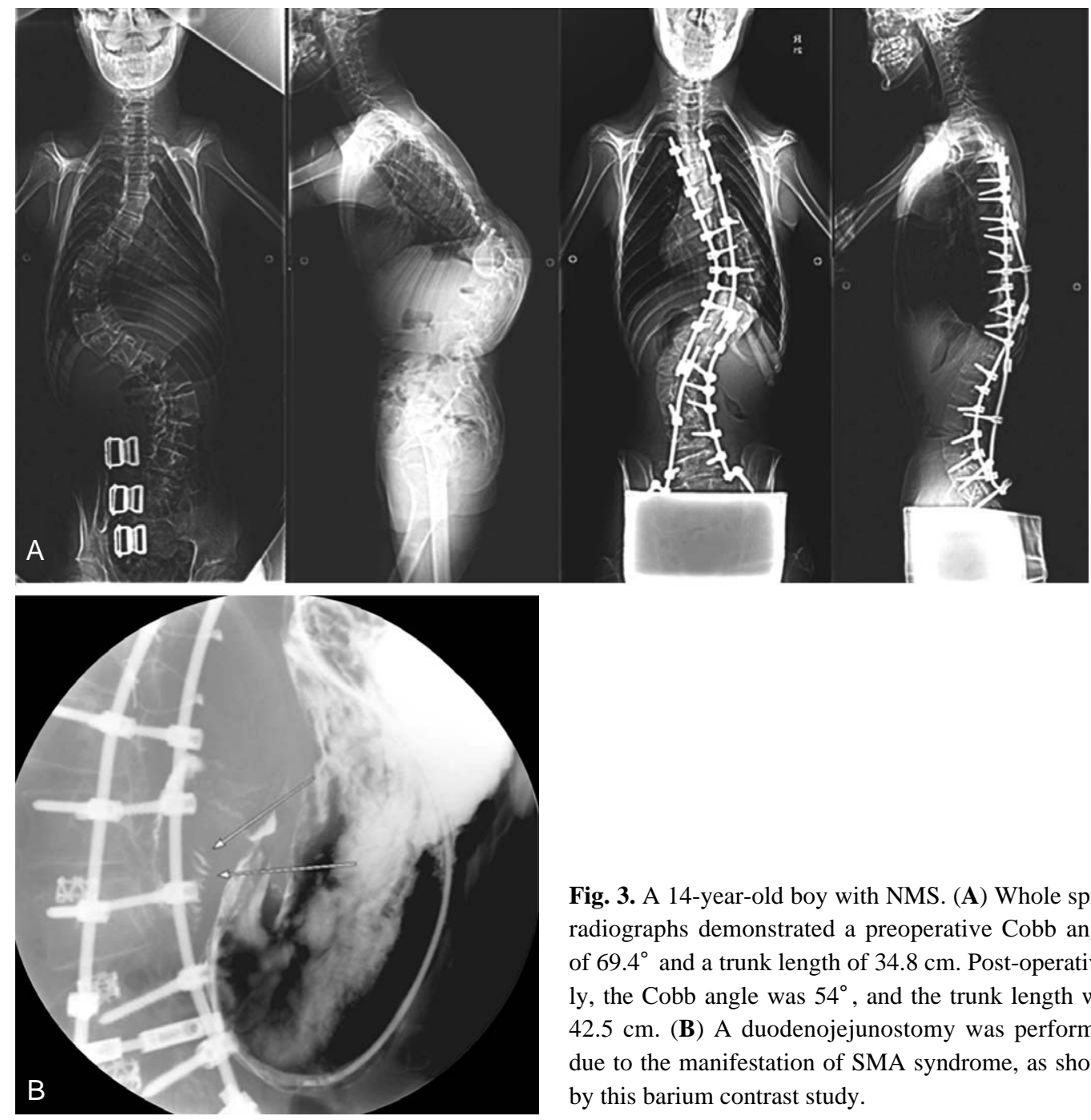

Fig. 3. A 14-year-old boy with NMS. (A) Whole spine radiographs demonstrated a preoperative Cobb angle of $69.4^{\circ}$ and a trunk length of $34.8 \mathrm{~cm}$. Post-operatively, the Cobb angle was $54^{\circ}$, and the trunk length was $42.5 \mathrm{~cm}$. (B) A duodenojejunostomy was performed due to the manifestation of SMA syndrome, as shown by this barium contrast study.

greater differences in BMI values were seen in NMS subjects. It is worth noting that the number of severely underweight subjects whose BMI fell below the 5th percentile was $32(29.4 \%)$ in the asymptomatic group and $6(66.7 \%)$ in the symptomatic group. The age- and sex-matched BMI percentile seems to be more predictive of SMA syndrome risk than is the absolute BMI.

The correctional change in curvature angles in the sagittal and coronal planes also plays a role in SMA syndrome risk. A greater angle of correction is accompanied by a greater increase in patient height. A previous study showed that the preoperative flexibility of the kyphosis angle, scoliosis angle, and the translation distance from the vertical center of the sacral line were important in predicting the occurrence of SMA syndrome ${ }^{12}$. However, one drawback of the study was the limitation of the subject pool to patients with adolescent idiopathic scoliosis.
Postoperative ileus shows many of the same clinical symptoms as SMA syndrome, thus making the clinical distinction difficult. General anesthesia, analgesics, electrolytic imbalance, and greater splanchnic nerve injury are all potential causes of post-operative ileus, which manifests in patients approximately 1 day post-operatively, and resolves anywhere from 3 to 5 days thereafter. Recent studies have noted the incidence of ileus to be between $3.5 \%$ and $6.1 \%{ }^{16,17}$. One of the greatest challenges in this study was to distinguish between SMA syndrome and ileus. The three subjects confirmed to have SMA syndrome showed symptoms at approximately one week post-operatively. However, a great number of patients showed similar symptoms around this period or immediately post-operatively. Of the total patient pool, 9 patients $(6.3 \%)$ were confirmed to have postoperative ileus, but that number increased to 13 patients (9\%) when those assumed to have the disorder were includ- 
Table 2. Statistical analysis of the entire subject pool according to the existence of symptom

\begin{tabular}{lccc}
\hline & Asymptomatic group $(\mathrm{n}=109)$ & Symptomatic group $(\mathrm{n}=9)$ & $\mathrm{p}$-value \\
\hline Weight $(\mathrm{kg})$ & $43.7 \pm 12.5$ & $36.2 \pm 7.8$ & 0.05 \\
Height $(\mathrm{cm})$ & $152.0 \pm 16.3$ & $160.6 \pm 7.2$ & 0.38 \\
BMI $\left(\mathrm{kg} / \mathrm{m}^{2}\right)$ & $18.5 \pm 3.5$ & $14.6 \pm 3.9$ & 0.001 \\
Age $($ years $)$ & $15.0 \pm 4.4$ & $15.9 \pm 3.6$ & 0.98 \\
Difference & $24.9 \pm 13.6$ & $23.4 \pm 9.1$ & 0.76 \\
$\quad$ in Cobb's angle $\left({ }^{\circ}\right)$ & $(52.7 \pm 24.2 \%)$ & $(51.5 \pm 14.3 \%)$ & $(0.88)$ \\
Difference & $2.3 \pm 2.1$ & $4.5 \pm 4.8$ & 0.08 \\
$\quad$ in trunk length $(\mathrm{cm})$ & $(7.0 \pm 7.2)$ & $(13.3 \pm 15.6)$ & $(0.12)$ \\
\hline
\end{tabular}

BMI: body mass index.

Table 3. Analysis of adolescent idiopathic scoliosis patients according to symptom existence

\begin{tabular}{lccc}
\hline & Asymptomatic group $(\mathrm{n}=76)$ & Symptomatic group $(\mathrm{n}=4)$ & $\mathrm{p}$-value \\
\hline Weight $(\mathrm{kg})$ & $47.8 \pm 10.5$ & $40.1 \pm 8.8$ & 0.26 \\
Height $(\mathrm{cm})$ & $160.5 \pm 8.1$ & $155.4 \pm 4.9$ & 0.37 \\
BMI $\left(\mathrm{kg} / \mathrm{m}^{2}\right)$ & $18.4 \pm 2.9$ & $17.2 \pm 3.6$ & 0.59 \\
Age $($ years $)$ & $15.8 \pm 3.4$ & $16.4 \pm 5.2$ & 0.74 \\
Difference & $27.0 \pm 12.6$ & $20.5 \pm 6.3$ & 0.48 \\
$\quad$ in Cobb's angle $\left({ }^{\circ}\right)$ & $(60.5 \pm 20.7 \%)$ & $(57.7 \pm 15.1 \%)$ & $(0.79)$ \\
Difference & $1.9 \pm 1.5$ & $0.8 \pm 0.3$ & 0.20 \\
$\quad$ in trunk length $(\mathrm{cm})$ & $(5.0 \pm 4.2 \%)$ & $(2.9 \pm 2.2 \%)$ & $(0.43)$ \\
\hline
\end{tabular}

BMI: body mass index.

Table 4. Analysis of neuromuscular scoliosis patients according to symptom existence

\begin{tabular}{lccc}
\hline & Asymptomatic group $(\mathrm{n}=33)$ & Symptomatic group $(\mathrm{n}=5)$ & $\mathrm{p}$-value \\
\hline Weight $(\mathrm{kg})$ & $40.2 \pm 11.6$ & $33.1 \pm 6.0$ & 0.10 \\
Height $(\mathrm{cm})^{*}$ & $144.3 \pm 13.1$ & $164.8 \pm 6.0$ & 0.002 \\
BMI $\left(\mathrm{kg} / \mathrm{m}^{2}\right)^{*}$ & $18.6 \pm 3.8$ & $12.5 \pm 2.8$ & 0.002 \\
Age $($ years $)$ & $14.4 \pm 5.3$ & $15.4 \pm 1.2$ & 0.73 \\
Difference & $19.9 \pm 11.8$ & $25.8 \pm 11.0$ & 0.34 \\
$\quad$ in Cobb's angle $\left(^{\circ}\right)$ & $(40.7 \pm 24.2 \%)$ & $(51.2 \pm 19.5 \%)$ & $(0.37)$ \\
Difference & $2.9 \pm 2.7$ & $7.0 \pm 5.1$ & 0.13 \\
$\quad$ in trunk length $(\mathrm{cm})$ & $(11.2 \pm 9.5 \%)$ & $(21.0 \pm 17.3 \%)$ & $(0.19)$ \\
\hline
\end{tabular}

BMI: body mass index.

ed. Constipation could also possibly be mistaken for SMA syndrome. We were unable to find previous elaboration on this disorder. However, patients undergoing correctional operations for scoliosis are prone to develop both pre-operative and post-operative constipation due to changes in environment and difficulty in movement. Teenage female patients and those with adolescent idiopathic scoliosis are particularly susceptible to constipation. Constipationinduced abdominal pain and distention were found in 12 patients $(8.3 \%)$ in this study. The time period during which patients began to suffer from symptoms was broad, running anywhere from 3 days to 10 days. But, patients with consti- pation exhibited abdominal pain without nausea or vomiting. Abdominal complaints in these patients resolved after defecation.

\section{Conclusions}

The incidence of SMA syndrome may be greater than previously thought, especially in patients with NMS. Close attention and careful scrutiny and testing should be exercised in patients with abdominal pain following correctional operations for scoliosis. In patients with a BMI under the 
5 th percentile, rigorous pre- and post-operative nutritional care and attention are important.

\section{REFERENCES}

1. Rokitansky C: Handbuch der pathologischen anotomie. Vienna, Branmiller and Siedel: 1842.

2. Tsirikos AI, Jeans LA: Superior mesenteric artery syndrome in children and adolescents with spine deformities undergoing corrective surgery. J Spinal Disord Tech 2005; 18: $263-271$.

3. Crowther MA, Webb PJ, Eyre-Brook IA: Superior mesenteric artery syndrome following surgery for scoliosis. Spine 2002; 27: E528-533.

4. Kennedy RH, Cooper MJ: An unusually severe case of the cast syndrome. Postgrad Med 1983; 59: 539-540.

5. Hod-Feins R, Copeliovitch L, Abu-Kishk I, et al: Superior mesenteric artery syndrome after scoliosis repair surgery: a case study and reassessment of the syndrome's pathogenesis. J pediatr orthop B 2007; 16: 345-349.

6. Shah MA, Albright MB, Vogt MT, Moreland MS: Superior mesenteric artery syndrome in scoliosis surgery: weight percentile for height as an indicator of risk. J pediatr orthop 2003; 23: 665-668.

7. Zhu ZZ, Qiu Y: Superior mesenteric artery syndrome following scoliosis surgery: its risk indications and treatment strategy. World J Gastroenterol 2005; 11: 3307-3310.

8. Bunch W, Delaney J: Scoliosis and acute vascular compression of the duodenum. Surgery 1970; 67: 901-906.

9. Stheneur C, Rey C, Pariente D, Alvin P: Acute gastric dilatation with superior mesenteric artery syndrome in a young girl with anorexia nervosa. Arch Pediatr 1995; 2: 973-976.

10. Hutchinson DT, Bassett GS: Superior mesenteric artery syndrome in pediatric orthopedic patients. Clin Orthop Relat Res 1990; 250: 250-257.

11. Vitale MG, Higgs GB, Liebling MS, Roth N, Roye DP Jr: Superior mesenteric artery syndrome after segmental instrumentation: a biomechanical analysis. Am J Orthop 1999; 8: 461-467.

12. Braun SV, Hedden DM, Howard AW: Superior Mesenteric Artery Syndrome Following Spinal Deformity Correction. J Bone Joint Surg Am 2006; 88: 2252-2257.

13. Boseker EH, Moe JH, Winter RB, Koop SE: Determination of "normal" thoracic kyphosis: a roentgenographic study of 121 "normal" children. J Pediatr Orthop 2000; 20: 796-798.

14. Willner S: A study of growth in girls with adolescent idiopathic structural scoliosis. Clin Orthop Relat Res 1974; 101: 129-135.

15. Munns SW, Morrissy RT, Golladay ES, Mckenzie CN: Hyperalimentation for superior mesenteric-artery (cast) syndrome following correction of spinal deformity. J Bone Joint Surg Am 1984; 66: 1175-1177.

16. Grossfeld S, Winter RB, Lonstein JE, Denis F, Leonard A, Johnson L: Complications of anterior spinal surgery in children. J Pediatr Orthop 1997; 17: 89-95.

17. Weis JC, Betz RR, Clements DH 3rd, Balsara RK: Prevalence of perioperative complications after anterior spinal fusion for patients with idiopathic scoliosis. J Spinal Disord 1997; 10: 371-375. 